\title{
Les diagnostics spécifiques de l'analyse des lasers X-U.V.
}

\author{
J. L. Bourgade, D. Desenne, J. P. Lebreton, M. Louis-Jacquet, C. Reverdin, R. Sauneuf, \\ D. Schirmann et P. Troussel
}

CEA/Centre d'Etudes de Limeil-Valenton, 94195 Villeneuve Saint Georges Cedex, France

A great diagnostic development effort has been performed since many years at the CEL-V in order to study UV and soft X-ray laser output characteristics. The main diagnostics used for this purpose are presented.

Le plein développement des expériences laser $\mathrm{X}$ a débuté au CEL-V par la mise en évidence d'une amplification par émission stimulée des ions néonoïde de germanium à la longueur d'onde de $230 \AA$ (1). Elles se sont ensuite poursuivies en collaboration avec les physiciens américains de Livermore (équipe de D. Matthews) (2) et ceux de l'équipe de P. Jaeglé . Le principe d'une expérience laser $X$ sur une installation laser de puissance est rappelé sur la figure ci-dessous

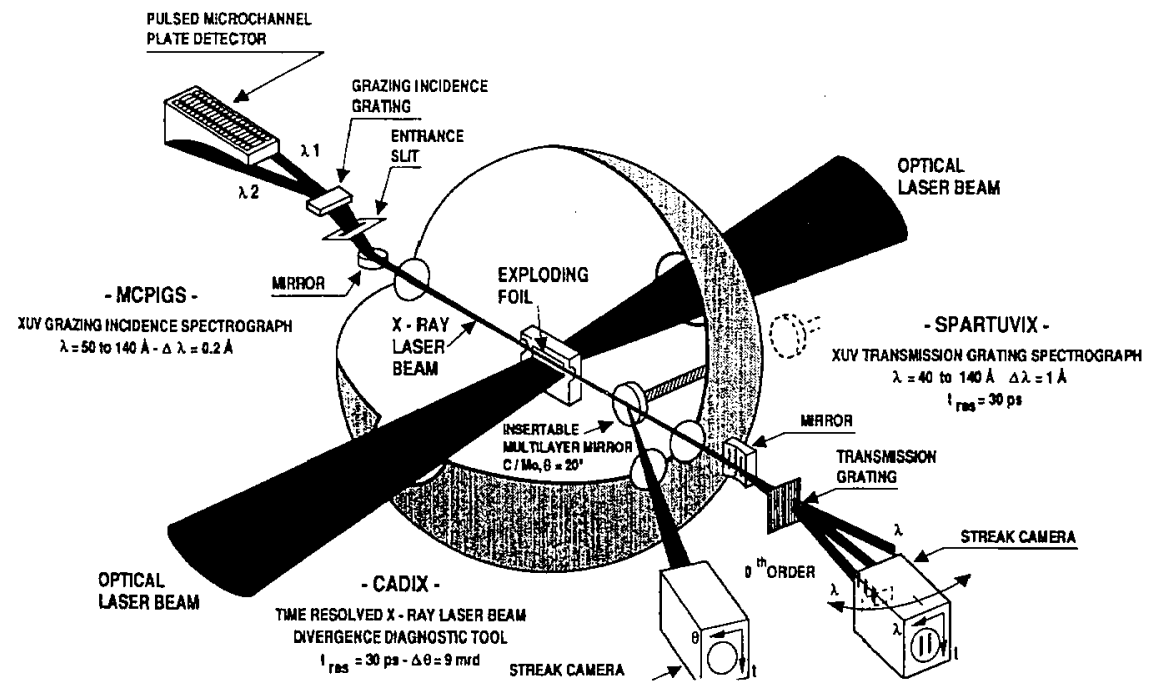

Dans le cas du laser PHEBUS (3) les deux faisceaux de puissance opposés ( $2200 \mathrm{~J} ; 0.53 \mu \mathrm{m} ; 500 \mathrm{ps}$ ) sont focalisés en un foyer linéaire réglable de 0 à $3 \mathrm{~cm}$. Le long de ces foyers est disposé une cible massive ou mince . Le plasma alors obtenu aux foyers de ce laser le plus puissant d'Europe peut donner lieu à une émission laser $\mathrm{X}$ suivant deux directions opposées. Cette émission peut être analysée suivant ces deux directions privilégiées par un ensemble de diagnostics spécifiques développés soit en propre au CEL-V soit en association (avec Livermore principalement). 
Ces diagnostics sont principalement des spectrographes X-U.V. à résolution temporelle; la signature d'une amplification étant souvent flagrante lorsqu'on examine l'évolution temporelle des raies lasante.

SPARTUVIX (4) est un spectrographe qui associe un miroir sphérique à un réseau en transmission. La détection, assurée par une caméra à balayage de fente confère à ce spectrographe une résolution temporelle de 30 ps. Le pas du réseau de $5000 \AA$ et les caractéristiques du miroir conduisent à une résolution spectrale inférieure à 1 A pour les émissions laser X.

MCPIGS (5) a été développé par Livermore pour l'analyse spécifique de l'émission laser $X$. C'est un spectrographe $X-U . V$. classique à réseau concave en incidence rasante qui disperse le spectre suivant le cercle de Rowland. L'originalité de ce spectrographe réside dans l'utilisation comme détecteur d'une galette courbe de microcanaux qui peut être pulsée par une haute tension assurant une fonction d'obturation rapide ( $200 \mathrm{ps}$ ). Le contraste entre une émission laser X brève et le reste de l'émission des raies du plasma de durée plus longue est alors notablement accru.

Ces spectrographes ont donné lieu au cours des différentes campagnes laser $X$ à une moisson de résultats importants: confirmant l'émission du germanium $(230 \AA)$, du sélénium $(210 \AA)$, du molybdène $(140 \AA)$ et de l'argent $(100 \AA)$ néonoïdes ou de l'oxygène hydrogénoïde $(100 \AA)$, établissant l'émission laser $X$ du strontium néonoide $(165 \AA)$ et analysant l'évolution temporelle de l'ytterbium nickeloïde $(80 \AA)$.

Ces spectrographes sont d'un encombrement important et nécessitent un alignement précis souvent long. Ils ne peuvent donc pas être momentanément déplacés pour laisser leur axe à un autre diagnostic.

CADIX (6) ne prend pas leur place mais intercepte momentanément le faisceau laser X à l'aide d'un miroir multicouche insérable sous vide. Le faisceau ainsi dévié peux être analysé par un diagnostic situé hors axe laser $\mathrm{X}$ et présent à leur coté . Comme premier diagnostic nous avons utilisé une caméra à balayage de fente pour analyser les caractéristiques angulaires d'émission d'un laser $X$ utilisant le schéma d'inversion collisionnel où la durée d'émission est plus brève que celle du reste du plasma. Le miroir multicouche plan sélectionne une bande spectrale autour de l'émission laser X de l'argent néonoïde à $100 \AA$. Un effet de réfraction important dû au gradient de densité du plasma a pu être mis en évidence: le faisceau laser $\mathrm{X}$ est dévié en dehors de l'axe du plasma lasant d'au moins 7 milliradians.

D'autres diagnostics peuvent être mis en place sur ce faisceau dévié comme par exemple un bolomètre (7) pour mesurer précisément l'énergie rayonnée par le laser $\mathrm{X}$ ou un analyseur de cohérence (8).

Cet effort instrumental important se poursuivra pour parfaire la connaissance des caractéristiques de ces laser X-U.V. en collaboration avec les équipes européennes et américaines sur les installations laser du CEL-V.

[1] LOUIS-JACQUET, M, et al., CR Acad. Sci. Paris. 306 série II (1988) 867

[2] KEANE, J.C, et al., Applied Physics B 50.(1990) 257

[3] THIELL, G, et al., Laser and Particle Beams. 6 (1988) 93

[4] BOURGADE, J-L, et al., Rev Sci. Instr. 59 (1988) 1846

[5] ECKART, M.J, et al., UCRL-52000-85-11 (1985) 25

[6] REVERDIN, C, et al., Rev Sci.Instr. à paraître Oct. 92

[7] à paraître, 3ème International Colloquium on X-ray lasers; SCHLIERSEE, mai 1992

[8] TREBES, J.E, Physical Review Letters. 68 (1992) 588 